\title{
DISCRIMINACIÓN Y ACTITUDES HACIA LA DIVERSIDAD AFECTIVO-SEXUAL: LA EXPERIENCIA DE LAS MUJERES.
}

\author{
DISCRIMINATION AND ATTITUDES TOWARDS SEXUAL-AFFECTIVE DIVERSITY:
}

THE EXPERIENCE OF WOMEN

Marta García Barba'
Naiara Martínez-Gómez
Cristina Giménez-García
Juan Enrique Nebot-García
Rafael Ballester-Arnal
Estefanía Ruiz-Palomino

Salusex-Unisexsida, Departamento de Psicología Básica, Clínica y de Psicobiología, Universitat Jaume I de Castellón

\section{RESUMEN}

A pesar de los cambios socioculturales, las personas LGTBI todavía deben hacer frente a ciertas dificultades. El objetivo de este estudio es explorar la discriminación y las actitudes hacia la diversidad afectivo-sexual en mujeres. 145 mujeres de entre 17 y 43 años $(M=29,63 ; D T=9,6)$, cumplimentaron un cuestionario ad hoc sobre discriminación y actitudes hacia el colectivo LGTBI. El 18,7\% de las mujeres no heterosexuales ha sufrido discriminación por orientación/identidad sexual. Más mujeres no heterosexuales que heterosexuales $(68 \%$ frente al $51,4 \%)$ han presenciado discriminación por estas cuestiones en su entorno. Encontramos ciertas actitudes negativas en ambos grupos, sobre todo en aquellos ítems relacionados con la expresión de género, la bisexualidad y ransexualidad. Estos hallazgos indican la necesidad de educar en diversidad y visibilizar aquellos colectivos que son más excluidos para lograr la equidad y el respeto.

Palabras Clave: Mujeres, discriminación, LGTBI, diversidad afectivo-sexual, actitudes. Cuenta con una ayuda predoctoral del programa ACIF/2018/241 de la Generalitat Valenciana. 


\section{ABSTRACT}

Despite sociocultural changes, LGTBI people still have to face certain difficulties. The purpose of this study is to explore discrimination and attitudes towards sexual-affective diversity in women. 145 women between 17 and 43 years old $(M=29,63 ; S D=9,6)$ completed an ad hoc questionnaire about discrimination and attitudes towards the LGTBI group. 18,7\% of non-heterosexual women have suffered sexual orientation / identity discrimination. More non-heterosexual women than heterosexuals $168 \% \mathrm{vs}$. $51,4 \%$ ) have witnessed discrimination by these issues in their environment. We found certain negative attitudes in both groups, especially in those items related to the expression of gender, bisexuality and transsexuality. These findings indicate the need to educate in diversity and make visible those groups that are most excluded to achieve equity and respect.

Keywords: Women, Discrimination, LGTBI, sexual-affective diversity, attitudes.

\section{SUMARIO}

1.- Introducción. 2.- Mirar de cerca (las contradicciones). Mayo del 68 y las mujeres. 3.- Los años setenta y ochenta. Entre la interseccionalidad y la identidad. 4.- ¿Éxito o precariedad? El feminismo ante neoliberalismo. 5.- Desafíos actuales. Feminismo poscrisis y recuperación de las formas políticas del feminismo de los setenta. 6.- Bibliografía.

\section{Introducción}

La American Psychological Association (APA, 2017) define la orientación sexual como una atracción emocional, romántica, sexual o afectiva duradera hacia otras personas. Esta atracción abarca todo un continuo que se extiende desde la heterosexualidad exclusiva hasta la homosexualidad exclusiva, incluyendo diversas formas de bisexualidad. No obstante, esto no siempre ha sido así. Para poder entender el concepto de diversidad sexual es necesario comprender, en líneas generales, cómo ha sido la evolución del mismo.

El término homosexualidad fue acuñado por primera vez en 1869 por Karl-Maria Kertbeny, sin embargo, no se popularizó hasta el año 1886 con la publicación del libro Psychopathia Sexualis de Richard Freiherr von Krafft-Ebing. Freud (1910) defendía que todas las personas partíamos de la bisexualidad, pero, posteriormente, íbamos desarrollando nuestra sexualidad, adoptando un comportamiento sexual normal, en el caso de resultar heterosexual, y una conducta desviada, en el caso de resultar homosexual. Posteriormente, Kinsey realizó dos de los estudios más completos sobre sexualidad-uno con hombres y otro con mujeres- cuyos resultados fueron plasmados en los libros Sexual Behaviour in the Human Male (1948) y Sexual Behaviour in the Human Female (1953). Los hallazgos, conocidos 
como el informe Kinsey, demuestran la prevalencia de la homosexualidad y bisexualidad en la sociedad, además de la existencia de deseos y experiencias sexuales homosexuales en la población heterosexual (Kinsey et al., 1948). Basándose en este estudio, Kinsey propuso que la orientación sexual debía ser entendida y evaluada como un continuo cuyos extremos los formarían la exclusiva heterosexualidad y la exclusiva homosexualidad pasando por diferentes grados de bisexualidad. Este estudio no estuvo exento de críticas, tanto por los hallazgos encontrados, como por las limitaciones que existían en el. Una de ellas fue que contemplaba la orientación sexual como una única dimensión.

Con el objetivo de subsanar esta limitación, en el año 1978 Klein elaboró la Klein Sexual Orientation Grid (KSOG). Esta escala proponía siete variables (atracción sexual, el comportamiento sexual, las fantasías sexuales, la preferencia emocional, la preferencia social, el estilo de vida y la auto-identificación o la auto-adscripción), dentro de la orientación sexual, que debían tenerse en cuenta al hablar de la misma. Además, subrayaba que la orientación sexual podía cambiar a lo largo del tiempo, por lo que se debe incluir el pasado, el presente y el ideal imaginado de cada persona. Estas aportaciones fueron de gran importancia para la bisexualidad, ya que evidenciaron su existencia y demostraron su alta prevalencia (Klein, 2012).

Tomando como punto de partida estas grandes aportaciones, diversos autores han realizado durante las últimas décadas diferentes investigaciones, con el objetivo de conocer la diversidad sexual en diferentes culturas y/o países y otorgarles mayor visibilidad a las orientaciones sexuales no heterosexuales. Sell et al. (1995) realizaron un estudio con población estadounidense, inglesa y francesa, en el que participaron un total de 2158 mujeres y 3931 hombres con edades comprendidas entre los 16 y los 50 años. Del total de las personas que participaron en el estudio, el $20,8 \% ; 16,3 \%$, y $18,5 \%$ de los hombres y el $17,8 \%, 18,6 \%$ y $18,5 \%$ de las mujeres evaluadas de Estados Unidos, Reino Unido y Francia respectivamente, afirmaron haber tenido comportamientos sexuales homosexuales y/o atracción sexual homosexual desde los 15 años. Respecto a los últimos 5 años, el 6,2\%, 4,5\% y $10,7 \%$ de los hombres y el 3,6\%,2,1\%, y 3,3\% de las mujeres encuestadas de Estados Unidos, Reino Unido y Francia, respectivamente, afirmaban haber mantenido relaciones sexuales con personas del mismo sexo en este lapso de tiempo. A nivel nacional, pocos son los estudios que encontramos al respecto. Uno de los más extensos y pioneros fue el realizado por Ballester y Gil (1995) en el que contaron con 1135 participantes de entre 14 y 70 años. En él encontraron que un 4,5\% de las personas evaluadas había mantenido relaciones sexuales con personas de su mismo sexo, el 7,7\% lo habían deseado en alguna ocasión y el 18,4\% había fantaseado sexualmente con personas de su mismo sexo. 
Pese a las evidencias sobre su considerable prevalencia en la sociedad, la orientación sexual no heterosexual no siempre ha estado bien vista y/o aceptada. De hecho, hasta el año 1973 la homosexualidad era considerada como una enfermedad mental, apareciendo hasta dicha fecha en el manual diagnóstico y estadístico de los trastornos mentales (DSM) como uno de los trastornos que lo componen. Otro ejemplo más es que no fue hasta el año 1990, cuando la Organización Mundial de la Salud (OMS) dejó de considerar la homosexualidad como una enfermedad. Lo cierto es que hasta lograr estos cambios se ha recorrido un camino extenso que no siempre ha sido progresivo. Según la Asociación Internacional de Lesbianas, Gays, Bisexuales, Trans e Intersex (ILGA, 2017), actualmente existen 72 estados en los que se criminalizan las relaciones sexuales entre personas del mismo sexo, siendo 27 de ellos los que criminalizan únicamente las relaciones entre hombres. Lo que apunta a que, en algunos países, a nivel legislativo hay distinciones de género entre las relaciones sexuales homosexuales donde la mujer no está reflejada en dichas leyes. No sabemos si puede ser por la falta de derechos que tienen las mujeres en algunos países, por la invisibilización de la sexualidad de las mujeres en general o de las relaciones lésbicas en particular o bien por una mayor aceptación de la homosexualidad de la mujer, lo que también podría darse por la objetización sexual de la mujer homosexual. Yost y McCarthy (2012), en su estudio encontraron que a algunas mujeres les cuesta distinguir si realizan estas conductas por su propio deseo o por agradar y satisfacer a otros hombres. Todo esto favorecería una mayor aceptación de la homosexualidad femenina.

A lo largo de la historia de España, nos hemos encontrado con una represión de la libertad sexual, como es el caso de la Ley de 1954 de Vagos y Maleantes o la Ley 16/1970 de Peligrosidad y Rehabilitación Social, entre otras. A partir de los años 80, España comenzó a promover los derechos de las personas LGTBI, entre la consecución de los derechos, destacamos la modificación de la Ley 77/1978 de Peligrosidad donde se excluyeron a las personas homosexuales de la misma y la reforma del Código Penal en la Ley Orgánica 10/1995, la cual establece el respeto y protección de la libre orientación sexual. Por otro lado, la Ley de Asilo (Ley 5/1984) permite conceder legalidad a los refugiados perseguidos por orientación sexual. Sin duda, uno de los mayores derechos obtenidos en España en lo referente a la comunidad LGTBI, en 2005, fue la aprobación del Proyecto de Ley de reforma de Código Civil (Ley 13/2005), donde se establece que todos los derechos de las parejas homosexuales son iguales a los de las parejas heterosexuales, permitiendo así el matrimonio entre personas del mismo sexo y la adopción, convirtiendo a España en el cuarto país del mundo que aceptaba estas uniones (Martín, 2016). Así mismo, en 2007 se aprobó la Ley de Identidad de Género (Ley 3/2007), la cual permite una reasignación de sexo y la rectificación en el registro en relación al sexo de las personas y el cambio de 
nombre. En la actualidad, a nivel autonómico (Navarra, País Vasco, Andalucía, Canarias, Madrid, Extremadura y Comunidad Valenciana) se está trabajando por la elaboración de leyes (Ley 8/2017) específicas que trabajan por los derechos de las personas transexuales y transgénero. Esto se produce no solo en lo que concierne a reconocer la identidad de las personas trans, sino a modificar todo lo referente a los plenos derechos, con la finalidad de conseguir una plena inclusión en la sociedad. A pesar de estos avances legislativos, todavía encontramos discriminación hacia el colectivo LGTBI en España. Según los datos ofrecidos por el Ministerio del Interior (2017) en el año 2017 hubo 270 delitos de odio por cuestiones de orientación e identidad sexual, 40 delitos más que los registrados en el año 2016.

En el resto de países, al igual que en España, todavía sigue existiendo discriminación hacia personas con una orientación sexual diferente a la heterosexual. Siendo los hombres quienes presentan actitudes menos favorables hacia el colectivo LGTBI (Costa y Davies, 2012; Fisher et al., 2017). Concretamente, las personas LGTBI se enfrentan a múltiples barreras y dificultades relacionadas con su orientación sexual, como el rechazo en el ámbito familiar y educativo (Fetner y Kush, 2008; Russell et al., 2011; Castillo, 2010). Así mismo algunas de las causas principales de los problemas que sufren estas personas se encuentra el estrés minoritario, debido al sentimiento de pertenencia a un grupo estigmatizado (López, 2008) y el miedo a ser rechazadas por el mero hecho de existir (Meyer, 2003), este miedo no necesariamente lo tienen que haber experimentado a través de situaciones discriminatorias, también se debe a aprendizajes derivados de acciones discriminatorias sobre otras personas (Grella et al., 2009).

En España, según estudio llevado a cabo por Marchueta (2014), entre los jóvenes homosexuales un $22 \%$ reconocen haber sufrido algún tipo de violencia física o psicológica tanto en el ámbito educativo (56\%), como en el ámbito familiar $(56 \%)$ así como en ambientes públicos (70\%). Por otro lado, en un estudio llevado a cabo en Estados Unidos, encontraron que el $44 \%$ de estudiantes homosexuales fueron agredidos físicamente a causa de su orientación sexual y un $85 \%$ había sufrido maltrato psicológico (Kosciw et al., 2008).

Debido a la discriminación hacia este colectivo que todavía persiste en la sociedad, las personas LGTBI experimentan consecuencias negativas en su salud mental, concretamente presentan mayor prevalencia de síntomas depresivos (Carroll, 2015; Espada et al., 2012) y ansiosos, baja autoestima (Marchueta, 2014; Martxueta y Etxeberria, 2014), mayor abuso de sustancias (Carroll, 2015; Huebner et al., 2015) e incluso ideación suicida (Capistrant y Nakash, 2018; Ybarra et al., 2015), entre otros.

Uno de los grandes problemas derivados de la discriminación y de las actitudes negativas hacia la homosexualidad, prevalentes en la sociedad, es lo que conocemos como homofobia interiorizada (Hernández y Aguilera, 2007). Esta situación se da cuando las 
personas LGB interiorizan los estereotipos negativos y las actitudes asociadas con su orientación sexual, por lo que acaban experimentando un gran estrés, tensión, vergüenza e incluso rechazo a la propia identidad (Meyer y Frost, 2013), lo que puede conllevar grandes consecuencias negativas en su salud mental (Walch et al., 2016).

Podemos pensar que, aunque es evidente que la discriminación hacia las personas pertenecientes al colectivo LGTBI sigue presente en la sociedad, cada vez es un colectivo más visible que puede encontrar apoyo dentro del mismo. A pesar de ello, no podemos obviar que es un colectivo muy diverso y por lo que, dentro del mismo, nos encontramos con grupos que reciben mayor discriminación. Empezando por las mujeres, décadas atrás, alrededor de los años 80-90 los bares y pubs "de ambiente" eran prácticamente los únicos sitios comunes donde conocer gente casi de forma clandestina. Sin embargo, algunos estudios muestran que las mujeres lesbianas $y / 0$ bisexuales perciben los espacios LGTBI como más amigables para los hombres (Corlover, 2013; Ferreira, 2011). Este es uno de los muchos ejemplos de cómo el sexismo se ve reflejado también en la vivencia de la sexualidad del propio colectivo, incluso en la forma de relacionarse. Las mujeres lesbianas y/o bisexuales deben romper con este sexismo predominante y transgredir los roles instaurados en la sociedad, lo que puede traducirse en una valoración negativa e incluso determinado tipo de sanciones (Cárdenas et al., 2012).

Otro de los colectivos más discriminados es el de las personas bisexuales quienes encuentran una doble discriminación, dada por parte de las personas heterosexuales, donde consideran a las personas bisexuales como promiscuas; como por parte de las personas gais/lesbianas, donde consideran a las personas bisexuales como indecisas, sintiendo que su orientación sexual no es tan válida como las otras (Doan Van et al., 2019).

Teniendo en cuenta la situación en la que se encuentran las mujeres no heterosexuales como colectivo expuesto a doble rechazo, debido a su identidad sexual y de género y debido a su orientación, el objetivo del presente estudio es explorar la experiencia de discriminación por motivos de orientación sexual en mujeres jóvenes y adultas, así como sus actitudes hacia la diversidad afectivo-sexual. Esperando que, pese a que la literatura ha demostrado que las mujeres presentan actitudes más favorables hacia la diversidad afectivo-sexual, encontraremos diferencias en función de la orientación sexual de las mismas, siendo las mujeres heterosexuales quienes muestren mayores prejuicios hacia el colectivo LGTBI. Además, las mujeres no heterosexuales habrán vivido más experiencias de discriminación que las heterosexuales, por lo que considerarán en mayor medida que todavía sigue existiendo discriminación hacia este colectivo tanto fuera como dentro del mismo. Finalmente, esperamos que, en general, encontraremos mayores prejuicios hacia el colectivo LGTBI relacionados con la expresión de género que con las prácticas sexuales en sí, tanto por parte de las mujeres heterosexuales como de las no heterosexuales. 


\section{MÉTODO \\ Participantes}

En el presente estudio participaron un total de 145 mujeres españolas con edades comprendidas entre los 17 y los 43 años $(M=29,63$; DT $=9,6)$. El $48,3 \%$ de las mujeres identificaba su orientación sexual como exclusivamente heterosexual, mientras que el resto $(51,7 \%)$ afirmaba que, en mayor o menor medida, se sentían atraídas también por otras mujeres, siendo algunas de ellas exclusivamente homosexuales. No encontramos diferencias significativas en cuanto a la media de edad en ambos grupos ( $t=0,98, p=, 922)$. El 57,9\% tenía pareja estable en el momento de la evaluación. Respecto a la formación académica, la mayoría $(84,8 \%)$ poseía estudios superiores, seguidas del 13,8\% que tenía estudios secundarios y un 1,4\% únicamente habría alcanzado estudios primarios. En cuanto a la situación laboral de cada una de ellas, un 5,5\% se encontraba en situación de desempleo, el 39,3\% era estudiante, el $11 \%$ combinaba trabajo con estudios y el $44,1 \%$ se encontraba activa a nivel laboral.

\section{Instrumentos}

El instrumento empleado en este estudio fue una entrevista estructurada, desarrollada ad hoc, en la que se recogía información sobre discriminación hacia el colectivo LGTBI y actitudes hacia el mismo, además de algunos datos sociodemográficos. Las preguntas referentes a la discriminación son 4 ítems de respuesta dicotómica en los que se exploraban las experiencias vividas de discriminación por orientación/identidad sexual y qué tipo de discriminación habían sufrido, haber presenciado algún tipo de discriminación por esta cuestión, y si consideran que hay discriminación tanto dentro como fuera del colectivo LGTBI. En el otro apartado de preguntas nos encontramos con una escala tipo Likert de 6 puntos ( $1=$ muy en desacuerdo y $6=$ muy de acuerdo) compuesta por 11 ítems directos que exploran las actitudes hacia el colectivo LGTBI. Entre estos ítems se observan algunos referentes a la presencia de ciertos estereotipos en cuanto a la conducta sexual (Ítem 2: Las personas bisexuales son más promiscuas, ítem 3: Las personas pansexuales son personas más sexualizadas, ítem 7: Las mujeres que tienen su primera experiencia con otra mujer a una edad adulta, lo hacen porque han tenido malas experiencias con los hombres e ítem 10: las personas no heterosexuales son un colectivo de riesgo para el $\mathrm{VIH}$ ) y otros relacionados con la expresión de género (ĺtem 4: Los hombres gais se cuidan más que los hombres heterosexuales, ítem 5: los hombres que muestran mucha pluma, en el fondo lo hacen para llamar la atención, ítem 6: los hombres gais son más femeninos que los hombres heterosexuales, ítem 8: las lesbianas 
que se muestran más masculinas es porque en el fondo quieren ser hombres). Cabe destacar que el número de ítems centrado en explorar las actitudes hacia cada orientación sexual (bisexual, gais, lesbianas) era equitativo. Siendo los ítems 1, 2 y 3 los que se relacionan con las actitudes hacia la bisexualidad, los ítems 3,4 y 5 los relacionados con las actitudes hacia los hombres homosexuales y los ítems 7,8 y 9 los asociados con las actitudes hacia las mujeres homosexuales. El resto de ítems eran más generales.

\section{Procedimiento}

Para llevar a cabo el presente estudio se elaboró una entrevista en formato online que fue difundida por diferentes redes sociales para acceder a una mayor variabilidad de población. En el mensaje de difusión del proyecto se explicaba el objetivo general de la evaluación, así como el carácter anónimo, voluntario y confidencial. Cuando las participantes daban su consentimiento informado, procedían a cumplimentarla.

Una vez recogidos los datos, se procedió a realizar los análisis estadísticos pertinentes mediante el programa estadístico SPSS versión 25.0. En primer lugar, se realizaron análisis descriptivos para caracterizar a la población participante. A continuación, se dividió en función de su orientación sexual obteniendo dos grupos: mujeres exclusivamente heterosexuales y mujeres no exclusivamente heterosexuales. Posteriormente, mediante el estadístico Chi cuadrado $\left(\chi^{2}\right)$ se calcularon las diferencias entre ambos grupos en cuanto a las vivencias de discriminación, las creencias sobre la existencia de la misma y las actitudes hacia ella. Finalmente se empleó el estadístico t de Student para comprobar si existían diferencias en las medias de las puntuaciones, obtenidas por cada uno de los dos grupos, en cuanto a las actitudes hacia gais, lesbianas y bisexuales por separado y las conductas sexuales y expresión de género del colectivo LGTBI.

\section{RESULTADOS}

En primer lugar, se analizó si las mujeres habían sufrido discriminación por cuestiones de identidad u orientación sexual. Un 18,7\% de las participantes cuya orientación sexual no es exclusivamente heterosexual ha sufrido discriminación por estas cuestiones, frente al $2,9 \%$ de las mujeres heterosexuales $\left(\chi^{2}=9,22 ; p=, 002\right)$. Un $43,75 \%$ de todas las mujeres que habían sufrido discriminación indicaron qué tipo de discriminación habían sufrido, siendo la más frecuente la verbal $(57,1 \%)$.

También se exploró si habían presenciado discriminación por cuestiones de identidad/orientación sexual en el entorno. Las mujeres heterosexuales reportaron haber presenciado estas situaciones en menor medida $(51,4 \%)$ que las mujeres con una orientación 
sexual diferente a la heterosexual $(68 \%)\left(\chi^{2}=4,14 ; p=, 042\right)$. En general, la mayoría de las mujeres consideran que existe discriminación hacia el colectivo $L G T B I+\left(\chi^{2}=2,51 ; p=, 113\right)$ sin embargo, encontramos diferencias significativas en cuanto a la creencia de la existencia de discriminación dentro del propio colectivo LGTBI+ entre los diferentes subgrupos, siendo las mujeres no exclusivamente heterosexuales las que más perciben la presencia de este tipo de discriminación $\left(\chi^{2}=17,93 ; p<, 001\right)$ (Ver tabla 1).

Tabla 1.

Diferencias en los porcentajes de creencias sobre la existencia de discriminación en mujeres en función de su orientación sexual.

\begin{tabular}{cccccc}
\hline & \multicolumn{2}{c}{ Heterosexual } & \multicolumn{2}{c}{$\begin{array}{c}\text { No exclusivamente } \\
\text { heterosexual }\end{array}$} & \multirow{2}{*}{$\chi^{2}(\mathrm{p})$} \\
\cline { 2 - 5 } & Sí (\%) & No (\%) & Sí (\%) & No (\%) & \\
\hline $\begin{array}{c}\text { ¿Consideras que existe } \\
\text { discriminación hacia el } \\
\text { colectivo LGTBI+? }\end{array}$ & 87,1 & 12,9 & 94,7 & 5,3 & 2,51 \\
& & & & & \\
\hline $\begin{array}{c}\text { ¿Consideras que existe } \\
\text { discriminación dentro del } \\
\text { propio colectivo LGTBl+ entre } \\
\text { los diferentes subgrupos? }\end{array}$ & 41,4 & 58,6 & 76 & 24 & $17,93)$ \\
\hline
\end{tabular}

En cuanto a las actitudes hacia el colectivo LGTBI+, no se encuentran diferencias significativas en las medias obtenidas en la totalidad del cuestionario, siendo un 19,23 la puntuación media obtenida por las mujeres heterosexuales y 19,30 la obtenida por las mujeres no heterosexuales $(t=-0,039 ; p=969)$. Por otro lado, se evaluaron las actitudes hacia el colectivo LGTBI+ entre las mujeres heterosexuales y las no exclusivamente heterosexuales, teniendo en cuenta la variabilidad dentro del propio colectivo. En la tabla 2 se detallan los porcentajes de mujeres que, en mayor o menor medida, están de acuerdo con las afirmaciones sobre este colectivo. Como se puede apreciar, no encontramos diferencias significativas, sin embargo, encontramos porcentajes relevantes a nivel cualitativo. Por ejemplo, tanto las mujeres heterosexuales como las no heterosexuales consideran en gran medida $160,9 \%$ y $59,2 \%$ respectivamente) que los hombres gais son más femeninos que los heterosexuales, y el $24,6 \%$ de las mujeres heterosexuales y el $32,9 \%$ de las mujeres no heterosexuales creen, en mayor o menor medida que, para estar con una mujer lesbiana masculina, estarían con un hombre. 
Tabla 2.

Diferencias en función de la orientación sexual en los porcentajes de respuestas de algo a completamente de acuerdo a los diferentes ítems.

\begin{tabular}{|c|c|c|c|}
\hline & Heterosexuales (\%) & $\begin{array}{c}\text { No } \\
\text { heterosexuales }(\%)\end{array}$ & $\chi^{2}(p)$ \\
\hline $\begin{array}{l}\text { 1. Las personas bisexuales son personas que } \\
\text { aún no tienen las cosas claras }\end{array}$ & 23,2 & 19,7 & $\begin{array}{c}0,26 \\
(, 613)\end{array}$ \\
\hline $\begin{array}{l}\text { 2. Las personas bisexuales son personas más } \\
\text { promiscuas. }\end{array}$ & 34,8 & 22,4 & $\begin{array}{c}2,75 \\
(, 097)\end{array}$ \\
\hline $\begin{array}{l}\text { 3. Las personas pansexuales son personas más } \\
\text { sexualizadas. }\end{array}$ & 36,2 & 38,2 & $\begin{array}{l}0,06 \\
(, 811)\end{array}$ \\
\hline $\begin{array}{l}\text { 4. Los hombres gais se cuidan más que los hom- } \\
\text { bres heterosexuales. }\end{array}$ & 55,1 & 59,2 & $\begin{array}{c}0,25 \\
(, 615)\end{array}$ \\
\hline $\begin{array}{l}\text { 5. Los hombres que muestran mucha pluma, en } \\
\text { el fondo, lo hacen para llamar la atención. }\end{array}$ & 36,2 & 40,8 & $\begin{array}{c}0,32 \\
(, 573)\end{array}$ \\
\hline $\begin{array}{l}\text { 6. Los hombres gais son más femeninos que los } \\
\text { heterosexuales. }\end{array}$ & 60,9 & 59,2 & $\begin{array}{c}0,04 \\
(, 839) \\
\end{array}$ \\
\hline $\begin{array}{l}\text { 7. Las mujeres que tienen su primera experien- } \\
\text { cia con otra mujer a una edad adulta, lo hacen } \\
\text { porque han tenido malas experiencias con los } \\
\text { hombres. }\end{array}$ & 34,8 & 40,8 & $\begin{array}{l}0,55 \\
(, 457)\end{array}$ \\
\hline $\begin{array}{l}\text { 8. Las lesbianas que se muestran más mascu- } \\
\text { linas, es porque en el fondo quieren ser hom- } \\
\text { bres. }\end{array}$ & 36,2 & 30,3 & $\begin{array}{l}0,58 \\
(, 446)\end{array}$ \\
\hline $\begin{array}{l}\text { 9. Para estar con una mujer lesbiana mascu- } \\
\text { lina, estaría con un hombre. }\end{array}$ & 24,6 & 32,9 & $\begin{array}{l}1,20 \\
(, 274)\end{array}$ \\
\hline $\begin{array}{l}\text { 10. Las personas no heterosexuales son un col- } \\
\text { ectivo de riesgo para el VIH. }\end{array}$ & 24,6 & 32,9 & $\begin{array}{r}1,20 \\
(, 274) \\
\end{array}$ \\
\hline $\begin{array}{l}\text { 11. Una persona transexual nunca llegará a ser } \\
\text { como alguien cis sexual. }\end{array}$ & 30,4 & 35,5 & $\begin{array}{c}0,42 \\
(, 515) \\
\end{array}$ \\
\hline \multicolumn{4}{|c|}{$\begin{array}{l}\text { En la tabla } 3 \text { se detallan las diferencias en las medias obtenidas al explorar las actitudes } \\
\text { acia cada uno de los colectivos. Para ello se calcularon las medias en cada una de las variables. } \\
\text { Como podemos observar, no encontramos diferencias significativas entre ambos grupos, desta- } \\
\text { cando que tanto las mujeres heterosexuales como las no heterosexuales puntuaron más alto en las } \\
\text { xctitudes negativas hacia los gais y más bajo en las actitudes negativas hacia personas bisexuales. }\end{array}$} \\
\hline
\end{tabular}


Tabla 3.

Diferencias en las medias entre mujeres heterosexuales y no heterosexuales en actitudes negativas hacia la población gay, bisexual y homosexual.

\begin{tabular}{cccc}
\hline & Heterosexuales & No heterosexuales & $t(\mathrm{p})$ \\
\hline $\begin{array}{c}\text { Actitudes negativas hacia } \\
\text { gais }\end{array}$ & 6,22 & 6,38 & $0,93(, 352)$ \\
\hline $\begin{array}{c}\text { Actitudes negativas hacia } \\
\text { bisexuales }\end{array}$ & 4,80 & 4,41 & $-0,30(, 764)$ \\
\hline $\begin{array}{c}\text { Actitudes negativas hacia } \\
\text { lesbianas }\end{array}$ & 5,07 & 5,17 & $-0,19(, 847)$ \\
\hline
\end{tabular}

Finalmente, se exploraron las actitudes hacia las personas LGTBI teniendo en cuenta si las actitudes discriminatorias estaban más relacionadas con las conductas sexuales o con la expresión de género. No se encontraron diferencias significativas entre las medias de cada uno de los grupos para cada variable. Sin embargo, cabe destacar que ambos grupos obtuvieron puntuaciones mayores en las creencias negativas sobre la expresión de género (tabla 4).

\section{Tabla 4.}

Diferencias en las medias entre mujeres heterosexuales y no heterosexuales en las creencias sobre la conducta sexual y la expresión de género dentro del colectivo LGTBI.

\begin{tabular}{lccc}
\hline & Heterosexuales & No heterosexuales & $\dagger(\mathrm{p})$ \\
\hline $\begin{array}{c}\text { Creencias negativas sobre } \\
\text { la conducta sexual }\end{array}$ & 6,51 & 6,49 & $0,04(, 970)$ \\
\hline $\begin{array}{c}\text { Creencias negativas sobre } \\
\text { la expresión de género }\end{array}$ & 9,67 & 9,84 & $-0,21(, 834)$ \\
\hline
\end{tabular}

\section{DISCUSIÓN Y CONCLUSIONES}

Este estudio tiene como objetivo explorar las vivencias de discriminación por identidad sexual y orientación sexual de las mujeres, heterosexuales y no heterosexuales, así como las actitudes de ambos grupos de mujeres hacia el colectivo LGTBI. En general, las mujeres evaluadas no muestran actitudes muy negativas hacia la diversidad afectivo-sexual, y muy pocas de ellas han vivido episodios de discriminación. Sin embargo, apenas encontramos 
diferencias entre las mujeres heterosexuales y aquellas cuya orientación sexual es diferente a la heterosexual en cuanto a las actitudes evaluadas. Esto puede ser debido a que, en general, los estudios indican que las mujeres tienen actitudes más favorables hacia la diversidad afectivo-sexual que los hombres (Carrera-Fernández, Lameiras-Fernández, Rodríguez-Castro y Vallejo-Medina, 2013). Esto podría explicar que las participantes, mujeres que en su mayoría tienen un nivel cultural elevado, presenten menos prejuicios hacia el colectivo LGTBI que los que encontrados en otros grupos de la sociedad.

Como hemos podido observar, los hallazgos indican que las mujeres que se identifican como no exclusivamente heterosexuales han sufrido en mayor medida que las mujeres heterosexuales algún tipo de discriminación, siendo la más frecuente la verbal. Autores como Hong et al. (2016) o Rankin et al. (2010) indican que actualmente es más frecuente la discriminación sutil que la manifiesta por lo que cabe esperar que la discriminación verbal sea superior a la física. Sin embargo, el hecho de que la discriminación más frecuente sea la sutil, podría dar lugar a que muchas situaciones se pasen por alto y no se perciban como discriminación. Este hecho, cabría tenerlo en cuenta tanto en próximas investigaciones como a nivel preventivo, ya que la discriminación sutil también puede causar malestar, incluso más que la discriminación directa (Molero et al., 2017). Esto también podría explicar por qué las mujeres evaluadas presenten mayor discriminación hacia el colectivo LGTBI por cuestiones de expresión de género que por cuestiones sexuales ya que este tipo de discriminación es mucho más sutil por lo que no está socialmente tan desaprobado.

Por otro lado, también se han observado cómo las mujeres con una orientación sexual diferente a la heterosexual percibían más discriminación tanto dentro como fuera del colectivo LGTBI. Aunque nuestros datos sugieran que el grupo hacia el que menos actitudes negativas hay es el de personas bisexuales, algunos estudios apuntan a que, dentro del propio colectivo LGTBI, encontramos ciertas actitudes discriminatorias, concretamente hacia personas bisexuales (Friedman, 2014). Según los hallazgos, podrían sentir que su orientación sexual no se considera válida ni por heterosexuales ni por personas lesbianas y gais (Doan Van et al., 2019). Además, estudios actuales apuntan que los individuos pertenecientes a minorías sexuales y de género emergentes (pansexual, demisexual, asexual, queer, questioning y transgénerol presentan mayor sintomatología depresiva y ansiosa que personas que se identifican como heterosexuales y/o cisgénero e incluso más que los que se identifican como gais o lesbianas (Borgogna et al., 2018). Por lo que consideramos que, en futuras líneas de investigación, sería conveniente explorar tanto las actitudes hacia las diferentes categorías, incluyendo las más tradicionales y las más recientes, como las experiencias de discriminación dentro de cada categoría. 
Este estudio no está exento de limitaciones, una de ellas es la muestra empleada. Una mayor cantidad y variabilidad de las personas participantes, podría favorecer la generalización de los resultados. De igual modo, cabría tener en cuenta que el instrumento es un autoinforme que podría prestarse a la deseabilidad social. Este tipo de herramientas de evaluación ha sido ampliamente empleado en investigación y resulta de gran utilidad para obtener una primera aproximación de la realidad que se pretende estudiar. En investigaciones posteriores y a partir de estos datos, será necesario elaborar y validar una escala que recoja las variables relevantes para el estudio y poder ofrecer unos datos más concisos, dando la oportunidad de poder replicar el estudio en otras poblaciones.

En cualquier caso, los hallazgos son relevantes en la medida en que suponen un acercamiento a la experiencia de las mujeres de la dimensión afectivo-sexual y, en particular, en un ámbito tan relevante como el de la discriminación y el estigma social. En este sentido, parece necesario que las estrategias de promoción de la salud psicosexual, incluyan esta perspectiva para así dar cabida a los grupos más invisibilizados dentro del propio colectivo y visibilizar aquellas actitudes y/o discriminaciones que puedan pasar desapercibidas, pero estén afectando a la calidad de vida de las personas que pertenecen al colectivo LGTBI. En este mismo sentido, también sería necesario el fomento de un empoderamiento entre las mujeres que también le otorgue importancia a la sexualidad y al disfrute, dejando atrás los prejuicios y la falsa asociación de la sexualidad en la mujer únicamente como medio reproductivo.

\section{REFERENCIAS}

American Psychological Association (APA) (2017). Orientación sexual e identidad de género. Disponible en: http://www.apa.org/centrodeapoyo/sexual.aspx (Fecha de consulta: 10/01/2019)

Asociación Internacional de Lesbianas, Gays, Bisexuales, Trans e Intersex (ILGA): CARRoll, Angus, y MENDOS, Lucas Ramón, Homofobia de Estado 2017: Estudio jurídico mundial sobre la orientación sexual en el derecho: criminalización, protección y reconocimiento (Ginebra: ILGA, mayo de 2017). Disponible en: http://ilga.org/ downloads/2017/ILGA_Homofobia_de_Estado_2017_WEB.pdf (Fecha de consulta: $10 / 01 / 20191$ 
RANKIN, Susan et al. (2010). State of higher education for lesbian, gay, bisexual y transgender people. Charlotte, NC: Campus Pride.

BalleSter, Rafael y María Dolores GIL (1995). «Homosexualidad: comportamientos deseos y fantasías» en Análisis y modificación de Conducta, № 21, pp. 264-284.

Borgogna, Nicholas Croft et al. (2018). «Anxiety and depression across gender and sexual minorities: Implications for transgender, gender nonconforming, pansexual, demisexual, asexual, queer, and questioning individuals» en Psychology of Sexual Orientation and Gender Diversity. № 6, Vol. 1, pp. 54-63.

Capistrant, Benjamin D., y Ora NaKash (2018). "Suicide Risk For Sexual Minorities in Middle and Older Age: Evidence from the National Study of Drug Use and Health» en The American Journal of Geriatric Psychiatry, doi: https://doi.org/10.1016/j. jagp.2018.12.023.

CÁrdenAS, Manuel et al. (2012). «Attitudes toward gay men and lesbians and their relationship with gender role beliefs in a sample of Chilean university students» en International Journal of Sexual Health, № 24, Vol. 3, pp. 226-236.

Carrera-Fernández, María Victoria et al. (2013). «Bullying among Spanish secondary education students: the role of gender traits, sexism, and homophobia» en Journal of interpersonal violence, $\mathrm{N}^{\circ} 28$, Vol. 14, pp. 2915-2940.

Carroll, Janell L (2015). Sexuality now: Embracing diversity (5th edition). EE UU: Cengage Learning.

CAstillo, Francisco Javier (2010). ¿Cómo perciben los jóvenes gays, lesbianas, bisexuales y transexuales el suicidio $y$, cuál es la conexión entre la identidad de género, la sexualidad y la conducta auto destructiva? en Índex de Enfermería, N 19, pp. 308309.

Corlouer, Melissa (2013). Quelle places pour lesbiennes? En Arnaud Alessandrin y Yves Ribaud (Eds), Géographie des homophobies. París: Editions Armand Colin.

COSTA, Pedro Alexandre y Michelle DAVIES (2012). «Portuguese adolescents' attitudes toward sexual minorities: Transphobia, homophobia, and gender role beliefs» en Journal of homosexuality, № 59, Vol. 10, pp. 1424-1442.

DOAN VAN, Emilie E. et al. (2019). «Perceived Discrimination, Coping Mechanisms, and Effects on Health in Bisexual and Other Non-Monosexual Adults» en Archives of sexual behavior, $N^{\circ} 48$, Vol. 1, pp. 159-174.

ESPADA, José Pedro et al. (2012). «Autoconcepto, ansiedad social y sintomatología depresiva en adolescentes españoles según su orientación sexual» en Ansiedad y Estrés, № 18 , Vol. 1, pp. 31-41. 
FerreirA, Eduarda. (2011) Geographies of (In)equalities: Space and Sexual Identities, En Rita Salvador, Firmino A., PONTE C. y Eduarda FerrelRa (eds), Proceedings of Geographies of Inclusion: Challenges and Opportunities. Lisboa: e-GEO Francisco, A. y Moliner, L. (2015). Descripción del modelo Encontrando Vidas a través de una investigación con mujeres migrantes lesbianas y bisexuales. Artículo inédito.

FEtNER, Tina y Kristin KUSH (2008). «Gay-straight alliances in high schools: Social predictors of early adoption» en Youth \& Society, № 40, Vol. 1, pp. $114-130$.

FISHER, Alessandra Daphne et al. (2017). «Who has the worst attitudes toward sexual minorities? Comparison of transphobia and homophobia levels in gender dysphoric individuals, the general population and health care providers» en Journal of endocrinological investigation, $\mathrm{N}^{\circ} 40$, Vol. 3, pp. 263-273.

Freud, Sigmund. (1910). «Three contributions to sexual theory» en New York Journal of Nervous and Mental Disorders, N 7, pp. 472-474.

Grella, Christine. E., et al. (2009). «Influence of gender, sexual orientation, and need on treatment utilization for substance use and mental disorders: Findings from the California Quality of Life Survey» en BMC psychiatry, N 9, Vol. 1, pp. 52-62.

Hernández, Celia y Eva María Agullera (2007). «La homofobia desde el Estado y la sociedad atenta contra los derechos humanos» en Revista d'Estudis de la Violencia, № 1, Vol. 3, pp. 1-22.

HONG, Jun Sung et al. (2016). «Ecological covariates of subtle and blatant heterosexist discrimination among $L G B Q$ college students» en Journal of Youth and Adolescence, $\mathrm{N}^{\circ}$ 45, Vol. 1, pp. 117-131.

Huebner, David M.; Thoma Brian C., y Torsten B. Neilands (2015). "School victimization and substance use among lesbian, gay, bisexual, and transgender adolescents» en Prevention Science, № 16, Vol. 5, pp. 734-743.

KINSEY, Alfred Charles et al. (1953). Sexual Behavior in the Human Female. Philadelphia. B. Saunders Company.

KINSEY, Alfred Charles, Pomeroy, Wardell B., y Clyde E. Martin (1948). Sexual behavior in the human male. Philadelphia: Saunders Company.

KLEIN, Fritz. (2012). La opción bisexual: Segunda edición. California, USA: American Institute of Bisexuality.

Kosciw, Joseph G.; Diaz, Elizabeth M. y Emily A. GREYLAK, (2008). The 2007 national school climate survey: the experiments of lesbian, gay, bisexual and transgender youth in our nation's school. New York: GLSEN.

KRAFFT-EBING, Richard Von (1886). Psychopathia sexualis (CG Chaddock, Trans.). Philadelphia: FA Davis. 
Ley $13 / 2005$, de 1 de julio, por la que se modifica el Código Civil en materia de derecho a contraer matrimonio. Boletín Oficial del Estado, núm. 157, de 2 de julio de 2005, pp. 23632 a 23634. Disponible en: https://www.boe.es/boe/dias/2005/07/02/ pdfs/A23632-23634.pdf (Fecha de consulta: 13/01/2019).

Ley $16 / 1970$, de 4 de agosto, sobre peligrosidad y rehabilitación social. Boletín Oficial del Estado, núm. 187, de 6 de agosto de 1970, páginas 12551 a 12557. Disponible en: https://www.boe.es/buscar/doc.php?id=BOE-A-1970-854. (Fecha de consulta: $13 / 01 / 2019)$.

Ley $3 / 2007$, de 15 de marzo, reguladora de la rectificación registral de la mención relativa al sexo de las personas. Boletín Oficial del Estado, núm. 65, de 16 de marzo de 2007, páginas 11251 a 11253 . Disponible en: https://www.boe.es/buscar/doc. php?id=BOE-A-2007-5585 (Fecha de consulta: 13/01/2019).

Ley $5 / 1984$, de 26 de marzo, reguladora del derecho de asilo y de la condición de refugiado. Boletín Oficial del Estado, núm. 74, de 27 de marzo de 1984, páginas 8389 a 8392. Disponible en: https://www.boe.es/buscar/doc.php?id=BOE-A-1984-7250. (Fecha de consulta: 13/01/2019)

Ley $77 / 1978$, de 26 de diciembre, de modificación de la Ley de Peligrosidad y Rehabilitación Social y de su Reglamento. Boletín Oficial del Estado núm. 10, de 11 de enero de 1979, páginas 658 a 659. Disponible en: https://www.boe.es/buscar/doc. php?id=BOE-A-1979-700 (Fecha de consulta: 13/01/2019).

Ley $8 / 2017$, de 7 de abril, integral del reconocimiento del derecho a la identidad y a la expresión de género en la Comunitat Valenciana. Diari Oficial de la Generalitat Valenciana, núm. 8019, de 11 de abril de 2017, pp. 12310 a 12333. Disponible en: https://www.dogv.gva.es/datos/2017/04/11/pdf/2017_3089.pdf. (Fecha de consulta: 13/01/2019).

Ley Orgánica 10/1995, de 23 de noviembre, del Código Penal. Jefatura del Estado Boletín Oficial del Estado, núm. 281, de 24 de noviembre de 1995 Referencia: BOE-A-1995-25444. Disponible en: https://www.boe.es/buscar/pdf/1995/BOEA-1995-25444-consolidado.pdf (Fecha de consulta: 13/01/2019).

LópEZ, Francisco José. (2008). La sexualidad en la adolescencia. Comunicación presentada en el XIX Congreso Nacional de la Sociedad Española de Medicina Adolescente de la AEP, Alicante.

MARChUETA, Aitor. (2014). «Consecuencias del bullying homofóbico retrospectivo y los factores psicosociales en el bienestar psicológico de sujetos $L G B »$ en Revista de Investigación Educativa, $\mathrm{N}^{\circ} 32$, Vol. 1, pp. 255-271. 
Martin, María (2016). "The Rights of Same-Sex Couples in Europe. Comparative Study» en Revista Española de Derecho Constitucional, N³6, Vol. 107, pp. $219-253$.

MarTXUetA, Aitor y Juan ETXEBERRIA. (2014). «Análisis diferencial retrospectivo de las variables de salud mental en lesbianas, gais y bisexuales (LGB) víctimas de bullying homofóbico en la escuela» en Revista de Psicopatología y Psicología Clínica, N ${ }^{\circ} 19$, Vol. 1, pp. 23-35.

MEYER, llan. H. (2003). «Prejudice, social stress, and mental health in lesbian, gay, and bisexual populations: conceptual issues and research evidence» en Psychological bulletin, $\mathrm{N}^{\circ} 129$, Vol. 5, pp. 674-697.

MeYer, Illan H., y David, M. Frost (2013). «Minority stress and the health of sexual minorities» en C. J. Patterson y A. R. D'Augelu (Eds.), Handbook of psychology and sexual orientation. New York, NY, US: Oxford University Press, pp. 252-266.

Ministerio del Interior (2017). Informe sobre la evolución de los incidentes relacionados con los delitos de odio en España. Disponible en: http://www.interior.gob.es/ documents/10180/7146983/ESTUDIO+INCIDENTES+DELITOS+DE+ODIO+ 2017+v3.pdf/5d9f1996-87ee-4e30-bff4-e2c68fade874 (Fecha de consulta: 09/01/2019).

Molero, Fernando et al. (2017). «Subtle and blatant perceived discrimination and well-being in lesbians and gay men in Spain: The role of social support.» en Psicothema, $\mathrm{N}^{\circ} 29$, Vol. 4, pp. 475-481.

RusselL, Stephen T. et al. (2011). «Lesbian, gay, bisexual, and transgender adolescent school victimization: Implications for young adult health and adjustment» en Journal of School Health, $\mathrm{N}^{\circ} 81$, Vol. 5, pp. 223-230.

Sell, Randall. L., Wells, James. A., y David Wypu. (1995). «The Prevalence of Homosexual Behavior and Attraction in the United States, the United Kingdom and France: Results of National Population-Based Samples» en Archives of Sexual Behavior, N ${ }^{\circ} 24$, pp. 234-248.

WALCH, Susan E. et al. (2016). «Discrimination, internalized homophobia, and concealment in sexual minority physical and mental health» en Psychology of Sexual Orientation and Gender Diversity, N ${ }^{\circ}$ 3, Vol. 1, pp. 37-48.

YBARRA, Michele. L., et al. (2015). «Understanding linkages between bullying and suicidal ideation in a national sample of LGB and heterosexual youth in the United States» en Prevention Science, N ${ }^{\circ} 16$, Vol. 3, pp. 451-462.

Yost, Megan R., y Lauren Mccarthy. (2012). «Girls gone wild? Heterosexual women's samesex encounters at college parties» en Psychology of Women Quarterly, No36, Vol. 1, pp. 7-24. 
